\title{
Long Non-Coding RNAs in Spinal Cord Injury
}

\author{
Rajan Jayanth ${ }^{1}$, Jean-Jacques Michaille ${ }^{2,3}$, Alexander A Efanov ${ }^{1}$, Hamdy Awad ${ }^{1}$ and Esmerina Tili ${ }^{1,3 *}$ \\ ${ }^{1}$ Department of Anesthesiology, The Ohio State University, USA \\ ${ }^{2}$ Université de Bourgogne-Franche Comté, France
}

${ }^{3}$ Department of Cancer Biology and Genetics, The Ohio State University Wexner Medical Centre and Comprehensive Cancer Centre, USA

*Corresponding author: Esmerina Tili, Department of Anaesthesiology, Wexner Medical Center, The Ohio State University, Columbus, OH 43210, USA.

Received Date: January 20, 2020

Published Date: January 30, 2020

\begin{abstract}
Non-coding RNAs whether short (such as microRNAs) or long $>200$ nucleotides, are implicated in a wide array of physiological and pathological processes including spinal cord injury. In this review, we will focus specifically on long non-coding RNAs and their involvement in spinal cord injury. We will first provide a background on the cellular and molecular mechanisms involved in spinal cord injury and emphasize the need to better understand these processes for developing effective therapeutics. Next, we will review the long non-coding RNAs reported to be changed by injury to the spinal cord, the study models and the techniques used, as well as the outcomes of injury when the expression of these transcripts is modified. We will finally discuss what can be done in the future for the experimental application of long noncoding RNAs in spinal cord injury.
\end{abstract}

\section{Introduction}

Spinal cord injury (SCI) and paralysis can be the result from: (I) a non-traumatic etiology such as viral infections (e.g. poliovirus can trigger poliomyelitis causing motor neuron death [1]), arthritis, and autoimmune disorders (e.g. multiple sclerosis in which white matter demyelination results in several neurological symptoms including gait ataxia, weakness, and optic neuritis [2]); (II) a traumatic injury (for example a motor vehicle accident); or (III) an ischemic event, caused by ischemic conditions imposed on the spinal cord during surgical interventions [3]. This review will only be focused on traumatic and ischemic spinal cord damage and longnoncoding RNAs.

\section{A-Spinal Cord Injury}

In traumatic SCI, the injury is sustained from a traumatic event (e.g. car accidents, falls, violence) [4] and can result in contusion [5], vertebral fracture and dislocation [6], or transection [7]. The damage caused by the initiating traumatic event is referred to as the primary injury; secondary injury refers to the biochemical reactions that follow the primary insult and exacerbate the injury to the spinal cord [8]. Secondary injury involves ionic imbalances and glutamate excitotoxicity, inflammation and inflammatory cytokine signaling, lipid peroxidation (LP), demyelination, axonal dieback, cell death, and gliosis and fibrosis [8]. The mechanical forces imparted by the primary injury disrupts the local vasculature and damages cells thus yielding higher amounts of extracellular debris including glutamate [8]; higher concentrations of extracellular glutamate dysregulates ionic homeostasis which ultimately promotes cell death [9]. Deleterious enzymes such as nitric oxide synthase and phospholipase A, activated by glutamate excitotoxicity [9], produce free radicals which initiates LP, a process that yields toxic 4-hydroxynonenal and 2-propenal [10] and produces protein adducts [11]. Free radicals generated from nitric oxide synthase and LP protein adducts also contribute to DNA damage which can result in neuronal death [12-14]. Vascular disruption results in neutrophil extravasation which, along with microglial activation, propagates inflammatory signaling through elevated inflammatory cytokine and chemokine secretion. Reports indicate that these processes exert some regenerative/neuroprotective signals at the synapse but due to the high intensity of the immune response at this point it is rather the detrimental signals that dominate the secondary effects in the injured spinal cord $[15,16]$. This inflammatory milieu further exacerbates the damage caused by the primary insult via activation of cell death programs (e.g. apoptosis, necroptosis), astrogliosis, glial scar formation, and extracellular matrix remodeling [8]. 
Ischemic SCI (ISCI) involves an ischemic etiology such as atherosclerotic disease, aortic pathologies, aortic surgery, and aortic grafting [17]. Damage to the spinal cord in ISCI is potentiated by ischemic/reperfusion (I/R) injury, which can occur during open surgical repair of thoracoabdominal aortic aneurysms (TAAA). In open surgery of TAAA, a cross-clamp is employed across the damaged aorta while the graft is implanted. Transient use of the cross-clamp induces ischemia of the spinal cord followed by blood flow reperfusion [18]. I/R of the spinal cord exacerbates damage as free radicals are generated and hyperemia contributes to spinal cord edema which subsequently induce neuronal death and LP [19]; concurrently, an inflammatory response in ISCI also contributes to damage through inflammatory cytokine secretion $[20,21]$. Studies of I/R injury in rats indicate that targeting components of the inflammatory cascade attenuated I/R injury and supported neurological improvement $[22,23]$. In addition, deletion of the proinflammatory microRNA miR-155 in mice reduced the incidence of paralysis after aortic cross-clamping in mice, further suggesting an inflammatory component to ISCI damage and paralysis after open aortic repair [21].

In the United States, the prevalence of SCI is reported to be 906 per million [24], and $90 \%$ of SCI cases have a traumatic etiology [4]. The impact of SCI upon patients and their families is tremendous: the damage caused by SCI can cause loss of function at and/or below the level of the neurological injury, patients who sustained the injury between the age of 25 and 34 years have a life expectancy of 38 years post-injury [25], and per individual economic burden can reach as high as $\$ 3.0$ million [26]. As of 2013, the only approved pharmaceutical therapy for SCI is methylprednisolone. Methylprednisolone possesses anti-inflammatory characteristics and attenuates LP yet it demonstrates varying efficacy in patients [27]. Accordingly, it is necessary to develop novel therapeutics aimed at attenuating neurological injury, promoting functional recovery, and bolstering endogenous regenerative efforts.

\section{B- Long noncoding RNAs}

Long non-coding RNAs (Lnc-RNAs), as the name implies, are RNA transcripts longer than 200 nucleotides that are for the most part not translated [28]. While there are also some Lnc-RNAs that have been shown to encode very short peptides [29], the research in this field is scarce [30]. Lnc-RNAs can be generated from the transcription of intergenic sequences (large intergenic noncoding RNA ie. Linc-RNA), from the antisense strand of recognized transcription units, from transcribed pseudogenes, from spliced-out introns, or from extraneous transcripts produced at transcription start sites [28]. Lnc-RNAs exert biologically relevant functions through a variety of mechanisms including as a scaffold for epigenetic regulators and as molecular sponges through RNARNA interactions [28]. The biological functions of Lnc-RNAs involve modulation of gene expression during development, the pathogenesis of cancer, and senescence [28,30]. The rest of this review will center on examining the role and potential therapeutic applicability of Lnc-RNAs in SCI.

\section{Lnc-RNAs in SCI}

As noted earlier, one mechanism by which Lnc-RNAs can exert their effects is through serving as a decoy for regulatory factors (sponging). Binding of a particular Lnc-RNA to a microRNA through complementary base recognition impairs the microRNA from binding to its targets; thus, the micrRNA is unable to reduce the expression of its protein-coding target transcripts, ultimately changing cellular homeostatic conditions. Lnc-RNA-microRNA interactions are the most extensively studied due to the possibility of predicting (in silico) Lnc-RNA-microRNA pairs.

In a contusion model of rat SCI, Lnc-RNA MALAT1, which was studied based on its roles in other diseases such as non-small cell lung carcinoma, was suggested to propagate inflammatory processes by interacting with miR-199b [31], a known regulator of the NF-kB inflammation pathway [32]. In fact, the study indicated that in vitro Lnc-RNA MALAT1 knockdown (KD) reduced tumor necrosis factor-alpha (TNF- $\alpha$ ) and interleukin 1 Beta (IL-1 $\beta$ ); these effects were reversed for the Lnc-RNA MALAT1 KD + miR-199b inhibition condition. Furthermore, Lnc-RNA MALAT1 (increased in SCI rats) is inversely correlated with miR-199b (decreased in SCI rats) expression and in vivo Lnc-RNA MALAT1 inhibition with lentiviral (LV)-siRNA-MALAT1 reduced inflammatory cytokine production and improved motor scores; these effects were reversed with antago-miR-199b treatment [31]. On the other hand, Qiao et al. proposed that Lnc-RNA MALAT1 has a beneficial effect after I/R SCI in rats by noting that Lnc-RNA MALAT1 overexpression in oxygen-glucose deprived neurocytes reduced miR-204, increased anti-apoptotic Bcl-2 and overall reduced apoptosis. These results translated to in vivo studies and I/R rats subjected to Lnc-RNA MALAT1 overexpression exhibited lower motor deficit indices while miR-204 overexpression reversed the anti-apoptotic effects of LncRNA MALAT1 overexpression [33]. We speculate that the contrasting effects of Lnc-RNA MALAT1 in these two studies could be due to the utilization of different SCI models, and the fact that Zhang et al. studied Lnc-RNA MALAT1 in microglial cell culture while the study conducted by Qiao et al. examined expression in neurocytes. These differential results raise a key point: it is important to acknowledge the cellular components involved in SCI and the cellular origin of genes/proteins, Lnc-RNAs, or microRNAs that are changed after SCI. In addition, considering the fact that microRNAs target different transcripts at different concentrations [34,35], depending on the level of expression of microRNAs and their interacting sponging Lnc-RNA, differential functional outcomes are expected. Another Lnc-RNA transcript involved in SCI is XIST. Studies have indicated that Lnc-RNA XIST has pro-apoptotic and pro-inflammatory role in SCI. Specifically, Zhao et al. indicates that in in vitro and in vivo models of SCI, Lnc-RNA XIST is upregulated and exerts deleterious effects through targeting the miR-27a/Smurf1 axis which was confirmed with a dual-luciferase assay and anti-miR-27a treatment in microglial cell culture; furthermore, in a contusion SCI rat model, Lnc-RNA XIST silencing using LV-sh-XIST infection decreased TNF- $\alpha$ and IL- 6 secretion, and Bax and cleaved-Caspase-3 expression, two 
genes involved in apoptosis [36]. Gu et al. suggested that Lnc-RNA $X I S T$ may sponge $m i R-494$, an oncomiR in hepatocellular carcinoma, which was confirmed using a luciferase assay; LV-sh-Lnc-RNA $X I S T$ administration in a contusion SCI rat model increased miR494 expression, activated the PI3K/Akt pathway, and improved functional recovery post-injury. The beneficial effects of LV-sh-LncRNA XIST administration were reversed when antago-miR-494 was also administered supporting the interaction between Lnc-RNA $X I S T$ and miR-494 [37]. It is interesting that this study found that reduction of expression of a Lnc-RNA using shRNA against XIST caused increased expression of its target microRNA. Our current understanding is that Lnc-RNAs bind and block the activity of a microRNA without necessarily changing the level of expression of this microRNA. Blocking the function of a microRNA does not necessary translate to decreased expression. Therefore, we caution that much more work should be done to better characterize and understand the outcomes of Lnc-RNA-microRNA interactions.

In an in vitro model of SCI, pc12 cells (neuronal cell culture) were more susceptible to $\mathrm{H}_{2} \mathrm{O}_{2}$-induced apoptosis and displayed higher protein levels of Bax, cleaved-Caspase-3 and 9, and lower Bcl-2 when Lnc-RNA $H 19$ was inhibited; Lnc-RNA H19 was studied because of seminal work demonstrating its putative roles in various cancers. The effect of Lnc-RNA H19 inhibition was attributed to higher miR-155 expression which targets FOXO1; miR-155 was demonstrated to be bound by Lnc-RNA H19. This experiment suggested a Lnc-RNA H19/miR-155/FOXO1 axis by which Lnc-RNA $H 19$ exerts neuroprotective effects; this paradigm is supported by the fact that the Lnc-RNA H19 inhibition + antisense-miR-155 condition reduced $\mathrm{H}_{2} \mathrm{O}_{2}$-induced injury [38]. In pc12 cells exposed to $\mathrm{H}_{2} \mathrm{O}_{2}$, Lnc-RNA Sox2ot is upregulated and Lnc-RNA Sox2ot inhibition attenuated injury induced by $\mathrm{H}_{2} \mathrm{O}_{2}$, which was attributed to the inverse correlation between LNC-RNA Sox2ot and miR-211 expression; miR-211 inhibitor treatment reversed the protective effects of Lnc-RNA Sox2ot inhibition. MCL-1 isoform 2 (MCL-1-i2) was identified as a direct target of $m i R-211$, and miR-211-mediated silencing of $M C L-1-i 2$ was determined to be responsible for the Akt/mTOR/p70S6k pathway activation [39]. Of note, the Akt/ mTor/p70S6k pathway is very well known for pro-survival signals, while the Mcl1 gene is part of proapoptotic machinery. In another in vitro model, pc12 cells exposed to LPS to mimic inflammatory conditions (LPS is a component of gram-negative bacterial cell wall) demonstrated attenuated NF-kB and p38 pathway activation and TNF- $\alpha$ and IL-6 secretion when Lnc-RNA Mirt2, which was found to upregulated in serum samples from SCI patients, was overexpressed. These results were attributed to Lnc-RNA Mirt2mediated downregulation of miR-429 [40]. Downregulation of PRDM5, which has numerous biological functions, by LNC-RNA DGCR5 or miR-130b-5p (which is sponged by LNC-RNA BDNF-AS) reduces neuronal apoptosis in vitro and in vivo [41,42]; in hypoxiatreated AGE1.HN (neural cell line [43]) and pc12 cell culture, Lnc-RNA DGCR5 overexpression reduced apoptosis while PRDM5 overexpression reversed these effects [41]. In hypoxia-treated AGE1.HN and pc12 cells, Lnc-RNA BDNF-AS KD reduced apoptosis
- an effect that was reversed with miR-130b-5p inhibition [42]. Following contusion SCI to rats, pcDNA-Lnc-RNA DGCR5 treatment improved motor scores [41]. Similarly, contusion SCI rats treated with LV-siRNA-Lnc-RNA BDNF-AS demonstrated increased miR130b-5p expression, and less PRDM5 and cleaved-Caspase3 expression, and fewer TUNEL positive cells [42]. In SY-SH-5Y cells (cell line derived from human hippocampal neurons) under oxygenglucose deprivation conditions, autophagy was induced by Lnc-RNA TCTN2 overexpression. Lnc-RNA TCTN2, which was downregulated after SCI in rats, was found to attenuate apoptosis and miR-216bmediated LC3II and Beclin-1 (both autophagy proteins) silencing; these effects were reversed with 3-methyladenine (an inhibitor of autophagy) or miR-216b mimic treatment. In a contusion model of rat SCI, overexpression of TCTN2 using pcDNA-Lnc-RNA TCTN2 resulted in better motor scores for contusion SCI rats thus demonstrating a beneficial role of autophagy after SCI [44].

Endogenous regenerative and repair efforts are also impacted by the ability of LNC-RNAs to serve as miR sponges. Neuronal proliferation supported by fibroblast growth factor 1 (FGF1) is impaired by miR-199a which was shown to target FGF1; Lnc-RNA MAP2K4 - miR-199a binding interaction, first identified by the authors through bioinformatic software, promotes proliferation and survival. The observed effects of Lnc-RNA MAP2K4 were reduced when miR-199a mimics were utilized or when $m i R-199 a$ binding sites on Lnc-RNA MAP2K4 were mutated [45]. LNC-RNA PTENP1, described as a tumor suppressor, was demonstrated by luciferase activity to regulate $m i R-21$ and $m i R-19 b$ which targets phosphatase and tensin homolog (PTEN) [46], a phosphatase that antagonizes Akt activation [47]. Wang et al. further reported that PTENP1 inhibition (using exosome-Lnc-RNA PTENP1 shRNA) may promote recovery after SCI as $m i R-21$ and $m i R-19 b$-mediated PTEN targeting reduced neuronal apoptosis and improved recovery in contusion SCI rats [46].

LNC-RNAs exerting biological functions through sense-antisense interactions are also relevant in the context of SCI. In a study concerning neuropathic pain, following induction of nerve injury via spinal nerve ligation in rats, Lnc-RNA Kcna2-AS was upregulated in the dorsal root ganglion [48]. Moreover, when Lnc-RNA Kcna2-AS was introduced in the spinal cord using AAV5-Lnc-RNA KCNA2-AS, it was demonstrated that this antisense transcript targets Kcna2 mRNA which resulted in increased dorsal root ganglion neuronal excitability and accordingly neuropathic pain symptoms. Finally, treatment with Kcna2 sense in spinal nerve ligation rats alleviated thermal, cold, and mechanical hypersensitivity [48].

Evidence indicates that several LNC-RNAs are implicated in the pathophysiology of SCI and its corresponding symptoms through complex mechanisms though the mechanisms that underly the functions of these Lnc-RNAs have not been completely elucidated. Bupivacanine-induced neurotoxicity in an in vitro explant model upregulated Lnc-RNA BDNF-AS, and Lnc-RNA BDNF-AS inhibition increased neurite outgrowth, reduced apoptosis, and increased Akt and TrkB phosphorylation [49]. The authors suggested that it 
is unlikely that Lnc-RNA $B D N F-A S$ exerts its deleterious effects by targeting BDNF, as the expression of BDNF and Lnc-RNA BDNF-AS are not consistently negatively correlated [49]. Lnc-RNA IGF2-AS inhibition in a dorsal root ganglion explant model also demonstrates neuroprotective effects following anesthetic treatment: LncRNA IGF2-AS inhibition promoted neurite outgrowth, attenuated apoptosis, and upregulated neurotrophins and growth factors following lidocaine treatment [50]. Astrogliosis following SCI is potentially linked to LNC-RNA SCIR1 based on a study conducted by Wang et al. LNC-RNA SCIR1 was found to be downregulated post contusion SCI in rats and knockdown of LNC-RNA SCIR1 in astrocyte culture prompted astrogliosis while Adm and $B m p 7 \mathrm{mRNAs}$ were upregulated and Wnt and SCNA mRNAs were downregulated. While bioinformatics predicted interactions between LNC-RNA SCIR1 and Adm, Bmp7, Wnt and SCNA mRNAs, the exact mechanism of LNC-RNA SCIR1-mediated regulation is yet to be delineated [51]. LNC-RNA SNHG16 was shown to be downregulated following $\mathrm{H}_{2} \mathrm{O}_{2}$ treatment; LNC-RNA SNHG16 overexpression ameliorated $\mathrm{H}_{2} \mathrm{O}_{2}$-induced injury in pc12 cells via upregulation of miR-423-5p which results in increased p-AMPK levels and decreased p-ERK1/2 levels; apoptosis and decreases in cell viability were reinstated with $m i R-423-5 p$ inhibition [52]. Finally, in contusion SCI mice, Lnc-RNA ZNF667-AS-1, studied for its role in cervical cancer, was overexpressed with LV-Lnc-RNA ZNF667-AS-1 which reduced JAK2, STAT3, and inducible nitric oxide synthase expression, thus displaying anti-inflammatory effects [53].

\section{Expression profiles of Lnc-RNAs in animal models of SCI}

Lnc-RNA expression profiling in SCI models is useful in identifying candidate Lnc-RNAs that may be involved in the pathophysiology of SCI. In a study conducted by Zhou et al,, microarray analysis was used to identify differentially expressed (DE) Lnc-RNAs in naïve rats compared to contusion SCI female Sprague-Dawley rats $2 \mathrm{~h}$ after injury [54]. In this study, 772 Lnc-RNAs were DE of which 528 Lnc-RNAs were upregulated and 244 LncRNAs were down-regulated compared to naïve rats [54]. Utilizing a near identical protocol, Shi et al. examined DE Lnc-RNAs 2 days post-injury; 3,193 DE Lnc-RNAs were identified of which 1,332 LncRNAs were upregulated and 1,861 Lnc-RNAs were downregulated compared to naïve rats [55]. There was no convergence between the top 10 upregulated Lnc-RNAs reported by Zhou et al. and the top 10 upregulated Lnc-RNAs reported by Shi et al.; there was no convergence between the top 10 downregulated Lnc-RNAs reported by Zhou et al. and the top 10 downregulated Lnc-RNAs reported by Shi et al. $[54,55]$. These differences are likely due to the different sampling times used in each experiment. In a study conducted by Cuevas-Diaz et al., female Sprague-Dawley rats were subjected to contusion SCI and DE (contusion SCI vs. sham operation) LncRNAs and protein-coding mRNAs were identified 1 month, 3 months, and 6 months post-injury using RNA-Seq [56]. The results indicated that 137 DE Lnc-RNAs were detected (120 upregulated, 17 downregulated compared to the sham group) 1 month postinjury, 239 DE Lnc-RNAs were detected (162 upregulated, 77 downregulated compared to the sham group) 3 months post- injury, and 179 DE Lnc-RNAs were detected (125 upregulated, 54 downregulated compared to the sham group) 6 months post-injury [56]. Hierarchical clustering indicated 5 clusters of DE Lnc-RNAs: the expression 79 Lnc-RNAs along with 790 protein-coding genes related to neuronal function gradually decreased as a function of time; the expression of 30 Lnc-RNAs along with 540 proteincoding genes enriched in cellular motility function genes gradually increased as a function of time; the expression of 46 Lnc-RNAs along with 880 protein-coding genes enriched in various focal adhesion genes increased rapidly at 3 months post-injury and increased until 6 months post-injury; the expression of 60 Lnc-RNAs and 1,420 protein-coding genes enriched in epigenetic regulation genes peaked at 1 month post-injury; the expression of 62 Lnc-RNAs along with 1,003 protein-coding genes enriched in inflammationassociated genes sharply increased at 1 month post-injury and retained high levels of expression over time [56]. Similarly, Ding et al. conducted a temporal analysis via microarray to study DE LncRNAs and protein-coding mRNAs in contusion SCI mice compared with sham-operated mice [57]. The results indicated that $345 \mathrm{DE}$ Lnc-RNAs were detected (164 upregulated, 181 downregulated compared to the sham group) 1 day post-injury, 502 DE Lnc-RNAs were detected (212 upregulated, 290 downregulated compared to the sham group) 3 days post-injury, 891 DE Lnc-RNAs were detected (326 upregulated, 565 downregulated compared to the sham group) 7 days post-injury and 181 DE Lnc-RNAs were detected (141 upregulated, 40 downregulated compared to the sham group) 21 days post-injury [57]. By examining correlations between DE protein-coding genes and Lnc-RNAs, Ding et al. were able to construct a dynamic Lnc-RNA-mRNA network with offers insight into Lnc-RNA function in SCI; this approach allowed identification of murine Lnc-RNAs that are mathematically associated with genes involved in the pathophysiology of SCI such as FGF1 and THBS1, which is reportedly involved AMPA receptor excitability, neuronal adhesion, and neurite outgrowth [57]. Both Cuevas-Diaz et al. and Ding et al. utilize Lnc-RNA - mRNA profiles to speculate the function of DE Lnc-RNA yet both articles also caution against drawing conclusions without further evidence [56,57]. Rather, the results of expression profiling studies serve as the foundation for more rigorous in vivo and in vitro studies designed to probe the function and mechanism of Lnc-RNAs in SCI. The latter two experiments raise two important points in Lnc-RNA expression following SCI: (1) Lnc-RNA signatures are highly variable and are functions of time and likely other experimental factors such as SCI model and must thus be understood in the context of experimental conditions, and (2) developing hypotheses regarding the functions of Lnc-RNA in SCI can be accomplished by computational and mathematical analysis of Lnc-RNA and protein-coding gene expression profiles.

\section{Conclusion}

Lnc-RNAs are RNA transcripts that could potentially exert many biologically relevant effects in numerous physiological and pathological processes including SCI. Recent studies indicate that modulating specific Lnc-RNAs in SCI models alters biochemical, 
histological, and neurological outcomes post-injury. We must emphasize however that the current knowledge regarding the roles of Lnc-RNAs in SCI is fragmented and must be consolidated and supplemented to develop future therapeutic applicability. One technical reason that has diminished our ability to rigorously study Lnc-RNAs is the lack of well-developed in situ hybridization techniques which is important in detecting the cellular localization of Lnc-RNAs. Moreover, Lnc-RNAs are poorly conserved across species rendering it difficult to identify their putative functions [58]. To improve our understanding of Lnc-RNAs in SCI, future studies may identify candidate Lnc-RNAs through RNA expression signatures. Such experiments may offer insight into novel mechanisms of LncRNA activity in SCI. For instance, although chromatin remodeling is implicated in SCI pathophysiology [59] and evidence indicates that Lnc-RNAs participate in the process of chromatin remodeling [58], Lnc-RNA-mediated chromatin remodeling has not been studied in SCI to our knowledge. Because there is a paucity of effective pharmacotherapies for SCI, it is important to study the roles of LncRNAs, their differential expression in response to cellular insult, and their cellular and subcellular localizations. In conclusion, LncRNAs hold potentials to be used for future therapeutics in SCI but much work remains to be done.

\section{Acknowledgement}

None.

\section{Conflict of Interest}

No conflict of interest.

\section{References}

1. S Ohka, C Nihei, M Yamazaki, A Nomoto (2012) Poliovirus trafficking toward central nervous system via human poliovirus receptordependent and -independent pathway. Front Microbiol 3.

2. DM Wingerchuk, CF Lucchinetti, JH Noseworthy (2001) Multiple Sclerosis: Current Pathophysiological Concepts. Laboratory Investigation 81(3): 263-281.

3. E Iseli, A Cavigelli, V Dietz, A Curt (1999) Prognosis and recovery in ischaemic and traumatic spinal cord injury: clinical and electrophysiological evaluation. Journal of Neurology, Neurosurgery \& Psychiatry 67(5): 567-571.

4. A Alizadeh, SM Dyck, S Karimi-Abdolrezaee (2019) Traumatic Spinal Cord Injury: An Overview of Pathophysiology, Models and Acute Injury Mechanisms. Front Neurol 10: 282.

5. G Ju, J Wang, Y Wang, X Zhao (2014) Spinal cord contusion. Neural Regen Res 9(8): 789-794.

6. I Koyanagi, Y Iwasaki, K Hida, M Akino, H Imamura, H Abe (2000) Acute cervical cord injury without fracture or dislocation of the spinal column. J Neurosurg 93(1): 15-20.

7. YH Cha, TH Cho, JK Suh (2010) Traumatic Cervical Cord Transection without Facet Dislocations-A Proposal of Combined HyperflexionHyperextension Mechanism: A Case Report. J Korean Med Sci 25(8): $1247-1250$.

8. A Alizadeh, SM Dyck, S Karimi-Abdolrezaee (2019) Traumatic Spinal Cord Injury: An Overview of Pathophysiology, Models and Acute Injury Mechanisms. Front Neurol 10.

9. Mark LP, Prost RW, Ulmer JL, Smith MM, Daniels DL, et al. (2001) Pictorial Review of Glutamate Excitotoxicity: Fundamental Concepts for Neuroimaging. American Journal of Neuroradiology 22(10): 1813-1824.
10. ED Hall, JA Wang, JM Bosken, IN Singh (2016) Lipid Peroxidation in Brain or Spinal Cord Mitochondria After Injury. J Bioenerg Biomembr 48(2): 169-174.

11. SG Codreanu, DC Liebler (2015) Novel Approaches to identify protein adducts produced by lipid peroxidation. Free Radic Res 49(7): 881-887.

12. Heneka MT, Löschmann PA, Gleichmann M, Weller M, Schulz JB, et al. (1998) Induction of Nitric Oxide Synthase and Nitric Oxide-Mediated Apoptosis in Neuronal PC12 Cells After Stimulation with Tumor Necrosis FActor- $\alpha /$ Lipopolysaccharide. Journal of Neurochemistry 71(1): 88-94.

13. S Dalleau, M Baradat, F Guéraud, L Huc (2013) Cell death and diseases related to oxidative stress:4-hydroxynonenal (HNE) in the balance. Cell Death Differ 20(12): 1615-1630.

14. LM Sayre, D Lin, Q Yuan, X Zhu, X Tang (2006) Protein adducts generated from products of lipid oxidation: focus on HNE and one. Drug Metab Rev 38(4): 651-675.

15. N Zhang, Y Yin, SJ Xu, YP Wu, WS Chen (2012) Inflammation \& apoptosis in spinal cord injury. Indian J Med Res 135(3): 287-296.

16. S David, JG Zarruk, N Ghasemlou (2012) Inflammatory pathways in spinal cord injury. Int Rev Neurobiol 106: 127-152.

17. MT Caton, JS Huff (2019) Spinal Cord Ischemia, in StatPearls, Treasure Island (FL): StatPearls Publishing.

18. H Awad, ME Ramadan, HF El Sayed, DA Tolpin, E Tili, et al. (2017) Spinal Cord Injury after Thoracic Endovascular Aortic Aneurysm Repair. Can J Anaesth 64(12): 1218-1235.

19. Wisselink W, Money SR, Crockett DE, Nguyen JH, Becker MO, et al. (1994) Ischemia-reperfusion injury of the spinal cord: Protective effect of the hydroxyl radical scavenger dimethylthiourea. Journal of Vascular Surgery 20(3): 444-450.

20. Ryu JH, Park JW, Hwang JY, Park SJ, Kim JH, et al. (2018) The attenuation of neurological injury from the use of simvastatin after spinal cord ischemia-reperfusion injury in rats. BMC Anesthesiology 18(1): 31.

21. Awad H, Bratasz A, Nuovo G, Burry R, Meng X, et al. (2018) MiR-155 deletion reduces ischemia-induced paralysis in an aortic aneurysm repair mouse model: Utility of immunohistochemistry and histopathology in understanding etiology of spinal cord paralysis. Annals of Diagnostic Pathology 36: 12-20.

22. L Fan, K Wang, Z Shi, J Die, C Wang, X Dang (2011) Tetramethylpyrazine protects spinal cord and reduces inflammation in a rat model of spinal cord ischemia-reperfusion injury. Journal of Vascular Surgery 54(1): 192-200.

23. Lu K, Cho CL, Liang CL, Chen SD, Liliang PC, et al. (2007) Inhibition of the MEK/ERK pathway reduces microglial activation and interleukin-1beta expression in spinal cord ischemia/reperfusion injury in rats. The Journal of Thoracic and Cardiovascular Surgery 133(4): 934-941.

24. A Singh, L Tetreault, S Kalsi-Ryan, A Nouri, MG Fehlings (2014) Global prevalence and incidence of traumatic spinal cord injury. Clin Epidemiol 6: 309-331.

25. M Wyndaele, JJ Wyndaele (2006) Incidence, prevalence and epidemiology of spinal cord injury: what learns a worldwide literature survey? Spinal Cord 44(9): 523-529.

26. H Krueger, VK Noonan, LM Trenaman, P Joshi, CS Rivers (2013) The economic burden of traumatic spinal cord injury in Canada. Chronic Dis Inj Can 33(3): 113-122.

27. Varma AK, Das A, Wallace G, Barry J, Vertegel AA, et al, (2013) Spinal Cord Injury: A Review of Current Therapy, Future Treatments, and Basic Science Frontiers. Neurochem Res 38(5): 895-905.

28. RW Yao, Y Wang, LL Chen (2019) Cellular functions of long noncoding RNAs. Nature Cell Biology 21(5): 542-551.

29. F Payre, C Desplan (2016) Small peptides control heart activity. Science 351(6270): 226-227.

30. M Montes, AH Lund (2016) Emerging roles of LncRNAs in senescence. FEBS J 283(13): 2414-2426. 
31. Zhou HJ, Wang LQ Wang DB, Yu JB, Zhu Y, et al. (2018) Long noncoding RNA MALAT1 contributes to inflammatory response of microglia following spinal cord injury via the modulation of a miR-199b/IKK $\beta / \mathrm{NF}$ KB signaling pathway. American Journal of Physiology-Cell Physiology 315(1): C52-C61.

32. Zhou HJ, Wang LQ, Xu QS, Fan ZX, Zhu Y, et al. (2016) Downregulation of miR-199b promotes the acute spinal cord injury through IKK $\beta-N F-k B$ signaling pathway activating microglial cells. Exp Cell Res 349(1): 60-67.

33. Y Qiao, C Peng, J Li, D Wu, X Wang (2018) LncRNA MALAT1 is Neuroprotective in a Rat Model of Spinal Cord Ischemia-Reperfusion Injury Through miR-204 Regulation. Curr Neurovasc Res 15(3): 211219.

34. E Tili, C M Croce, JJ Michaille (2009) miR-155: on the crosstalk between inflammation and cancer. Int Rev Immunol 28(5): 264-284.

35. JJ Michaille, H Awad, EC Fortman, AA Efanov, E Tili (2019) miR-155 expression in antitumor immunity: The higher the better? Genes Chromosomes Cancer 58(4): 208-218.

36. Zhao Q, Lu F, Su Q, Liu Z, Xia X, et al. (2020) Knockdown of long noncoding RNA XIST mitigates the apoptosis and inflammatory injury of microglia cells after spinal cord injury through miR-27a/Smurf1 axis. Neuroscience Letters 715: 134649 .

37. S Gu, R Xie, X Liu, J Shou, W Gu, X Che (2017) Long Coding RNA XIST Contributes to Neuronal Apoptosis through the Downregulation of AKT Phosphorylation and Is Negatively Regulated by miR-494 in Rat Spinal Cord Injury. International Journal of Molecular Sciences 18(4): 732.

38. R Li, F Yin, Y Guo, K Zhao, Q Ruan, Y Qi (2017) Long non-coding RNA H19 protects PC-12 cells from hydrogen peroxide-induced injury by targeting miR-155 in spinal cord injury. International Jorunal of Clinical and Experimental Pathology 10(6): 14.

39. D Yin, X Zheng, J Zhuang, L Wang, B Liu, et al. (2018) Downregulation of long noncoding RNA Sox2ot protects PC-12 cells from hydrogen peroxide-induced injury in spinal cord injury via regulating the miR-211-myeloid cell leukemia-1 isoform2 axis. Journal of Cellular Biochemistry 119(12): 9675-9684.

40. H Li, Y Xu, G Wang, X Chen, W Liang, H Ni (2019) Long non-coding RNA Mirt2 relieves lipopolysaccharide-induced injury in PC12 cells by suppressing miR-429. J Physiol Biochem 75(3): 403-413.

41. Huafeng Zhang, Wengang Wang, Ning Li, Peng Li, Ming Liu, et al. (2018) LncRNA DGCR5 suppresses neuronal apoptosis to improve acute spinal cord injury through targeting PRDM5. Cell Cycle 17(16): 1992-2000.

42. Zhang H, Li D, Zhang Y, Li J, Ma S, et al. (2018) Knockdown of LncRNA BDNF-AS suppresses neuronal cell apoptosis via downregulating miR130b-5p target gene PRDM5 in acute spinal cord injury. RNA Biol 15(8): 1071-1080.

43. Eva Schräder, Sebastian Scholz, Jens Niklas, Alexander Rath, Oscar Platas Barradas, et al., (2011) Characterisation of cultivation of the human cell line AGE1.HN.AAT. BMC Proc 5(8): P87.

44.X Ren, C Wan, Y Niu (2019) Overexpression of LncRNA TCTN2 protects neurons from apoptosis by enhancing cell autophagy in spinal cord injury. FEBS Open Bio 9(7): 1223-1231.
45. H Lv (2017) LncRNA-Map2k4 sequesters miR-199a to promote FGF1 expression and spinal cord neuron growth. Biochemical and Biophysical Research Communications 490(3): 948-954.

46. Z Wang, Y Song, X Han, P Qu, W Wang (2020) Long noncoding RNA PTENP1 affects the recovery of spinal cord injury by regulating the expression of miR-19b and miR-21. Journal of Cellular Physiology 235(4): 3634-3645.

47. TO Chan, SE Rittenhouse, PN Tsichlis (1999) AKT/PKB and Other D3 Phosphoinositide-Regulated Kinases: Kinase Activation by Phosphoinositide-Dependent Phosphorylation. Annu Rev Biochem 68(1): 965-1014.

48. Xiuli Zhao, Zongxiang Tang, Hongkang Zhang, Fidelis E Atianjoh, Jian-Yuan Zhao, et al. (2013) A long noncoding RNA contributes to neuropathic pain by silencing Kcna 2 in primary afferent neurons. Nature Neuroscience 16(8): 1024-1031.

49. Y Zhang, L Yan, Y Cao, G Kong, C Lin (2016) Long noncoding RNA BDNF-AS protects local anesthetic induced neurotoxicity in dorsal root ganglion neurons. Biomedicine \& Pharmacotherapy 80: 207-212.

50. X Zhang, K Chen, C Song, C Song (2016) Inhibition of long non-coding RNA IGF2AS has profound effect on inducing neuronal growth and protecting local-anesthetic induced neurotoxicity in dorsal root ganglion neurons. Biomedicine \& Pharmacotherapy 82: 298-303.

51. J Wang, B Hu, F Cao, S Sun, Y Zhang, et al. (2015) Down regulation of lncSCIR1 after spinal cord contusion injury in rat. Brain Research 1624: 314-320.

52. H Liu, B Chen, Q Zhu (2019) Long non-coding RNA SNHG16 reduces hydrogen peroxide-induced cell injury in PC-12 cells by up-regulating microRNA-423-5p. Artificial Cells, Nanomedicine, and Biotechnology 47(1): 1444-1451.

53. H Liu, B Chen, Q Zhu (2019) LncRNA ZNF667-AS1 promotes SCI recovery. Artifical Cells, Nanomedicine, and Biotechnology 47(1): 7.

54. Zhou H, Shi Z, Kang Y, Wang Y, Lu L, et al. (2018) Investigation of candidate long noncoding RNAs and messenger RNAs in the immediate phase of spinal cord injury based on gene expression profiles. Gene 661: $119-125$.

55. Shi Z, Ning G, Zhang B, Yuan S, Zhou H, et al. (2019) Signatures of altered long noncoding RNAs and messenger RNAs expression in the early acute phase of spinal cord injury. J Cell. Physiol 234(6): 8918-8927.

56. Duran RC, Yan H, Zheng Y, Huang X, Grill R, et al. (2017) The systematic analysis of coding and long non-coding RNAs in the sub-chronic and chronic stages of spinal cord injury. Sci Rep 7: 41008.

57. Y Ding, Z Song, J Liu (2016) Aberrant LncRNA Expression Profile in a Contusion Spinal Cord Injury Mouse Model. Biomed Res Int 2016.

58. A Saxena, P Carninci (2011) Long non-coding RNA modifies chromatin. Bioessays 33(11): 830-839.

59. EM York, A Petit, AJ Roskams (2013) Epigenetics of Neural Repair Following Spinal Cord Injury. Neurotherapeutics 10(4): 757-770, 